\title{
Psychosocial Factors Predict COVID-19 Vaccine Side Effects
}

\author{
Andrew L. Geers ${ }^{\mathrm{a}}$ Kelly S. Clemens ${ }^{\mathrm{a}}$ Kate Faasse ${ }^{\mathrm{b}}$ Ben Colagiuri ${ }^{\mathrm{c}}$ \\ Rebecca Webster $^{d}$ Lene Vase $^{\mathrm{e}}$ Mette Sieg $^{\mathrm{e}}$ Emily Jason $^{\mathrm{a}}$ Luana Colloca $^{f}$

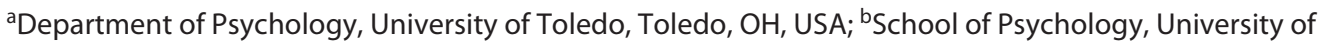 \\ New South Wales, Sydney, NSW, Australia; 'School of Psychology, University of Sydney, Sydney, NSW, Australia; \\ ${ }^{\mathrm{d} D e p a r t m e n t}$ of Psychology, University of Sheffield, South Yorkshire, UK; 'Department of Psychology and \\ Behavioural Sciences, Aarhus University, Aarhus, Denmark; 'Departments of Pain Translational Symptoms Science, \\ University of Maryland, Baltimore, MD, USA
}

Dear Editor,

Recently, Cosci and Guidi [1] proposed a framework based on the concept of illness behaviour [2] to explain the psychosocial consequences of the COVID-19 pandemic in terms of occurrence of psychosomatic syndromes and distinct manifestations of the large spectrum of anxiety, phobias, hypochondriasis, and illness denial. Furthermore, beyond psychopathology, Colloca et al. [3] documented that individuals suffering from chronic pain experienced a worsening in depression and anxiety paralleled by an improvement of pain severity and coping during a 21-day stay-at-home period during the pandemic.

Can psychosocial approaches such as that of Cosci and Guidi [1] and Colloca et al. [3] be extended to explain, at least in part, COVID-19 vaccine reactogenicity? Localized and systemic side effects are frequently reported following coronavirus disease 2019 (COVID-19) vaccinations. Although primarily non-serious, side effects can make vaccination a disagreeable experience and side effect concerns are among the most common reasons for vaccine hesitancy [4]. For example, a recent US poll found $90 \%$ of COVID-19 vaccination refusers were more worried about vaccine side effects than COVID-19 infection [5].

karger@karger.com

(c) 2021 S. Karger AG, Basel

www.karger.com/pps

Karger!
Rief [6] posited that reactogenicity to COVID-19 vaccines and vaccine hesitancy might be influenced by psychosocial factors, particularly nocebo effects. Nocebo effects occur when negative expectations and related factors (e.g., worry, depressive symptoms) trigger or exacerbate negative symptoms [7]. Nocebo effects go beyond response bias and have neurobiological signatures [7]. Many symptoms reported as COVID-19 vaccine side effects, including pain, fatigue, nausea, and headache, are known to be sensitive to nocebo effects. Thus, psychosocial factors, such as expectations, emotions, and attitudes, may modulate psychological or physical side effect responses to COVID-19 vaccines. For example, the vaccine may cause fatigue, but this symptom can be amplified by the individual's expectations and selective attention towards this potential side effect. Also, side effects such as headache emerged in $18-34 \%$ of placebo group participants in Pfizer-BioNTech vaccine trials [8], suggesting non-pharmacological causes. Therefore, nocebo effects may worsen side effects related to the COVID-19 vaccines and thereby decrease the likelihood of vaccination by affecting social communication and decision-making processes. 
Yet, the relationship between nocebo-related factors and COVID-19 vaccine reactogenicity has not been tested. The current study addressed this gap and aimed to identify nocebo-related predictors of COVID-19 vaccine side effects that can be targeted to reduce their occurrence and, consequently, vaccine hesitancy.

A preregistered (Open Science Framework, https:// osf.io/r6utm) prospective longitudinal study was conducted with a US national sample of individuals aged $\geq 18$ years. Participants were recruited through the online recruitment platform Prolific. The study was approved by the local Institutional Review Board (Protocol: 300993 UT). Participants received monetary compensation and gave electronic informed consent prior to participation. The study consisted of a pre-vaccination survey (Survey 1 ) and a post-vaccination survey (Survey 2). Eligibility criteria for Survey 1 included being non-vaccinated, and the survey was completed by 1,561 individuals. Survey 1 was open from April 15-28, 2021 and assessed noceborelated factors, including expectations for seven side effects identified in COVID-19 vaccine randomized clinical trials and publicized by the Centers for Disease Control and Prevention (CDC, https://www.cdc.gov/ coronavirus/2019-ncov/vaccines/expect/after.html) (i.e., pain at injection site, fever, chills, headache, joint pain, nausea, fatigue). In Survey 1, individuals also completed self-report measures of baseline systemic symptoms and sociodemographic questions. Eligibility for Survey 2 included both completion of Survey 1 and full vaccination between completion of Survey 1 and Survey 2 . Survey 2 was completed by 551 vaccinated individuals (56.4\% Pfizer-BioNTech, 33.1\% Moderna, and 10.5\% Janssen/Johnson \& Johnson), was open from May 21 to July 19, 2021, and assessed experience of the same seven CDC advertised vaccine side effects measured in Survey 1. Full vaccination status was not available for non-responders of Survey 2. Yet, of the 1,561 individuals completing Survey 1, 585 participants reported to Prolific having received at least one COVID-19 vaccine dose by the close of Survey 2, providing an approximated $94 \%$ retention rate. The 551 participants $\left(52.7 \%\right.$ women; $\mathrm{M}_{\text {age }}$ $=31.66 ; \mathrm{SD}_{\text {age }}=11.05$; age range $\left.=18-71\right)$ represented 48 of the 50 United States, were 69\% White, 12\% Hispanic, $49.7 \%$ with a bachelor's degree education or higher, $45.3 \%$ with an income above USD 60,000, and $19.4 \%$ reported having contracted COVID-19. Vaccination status was substantiated by Prolific's recruitment management system for Survey 1, self-reports at the start of both surveys, and for Survey 2 , information listed on participants' CDC vaccination card.
The relationship between nocebo-related factors and COVID-19 vaccine reactogenicity was tested with multivariable Poisson regression. Nagelkerke's pseudo- $R^{2}$ [9] was used to quantify variance accounted for by predictors, as traditional $R^{2}$ is not appropriate for count-based regression models. We found that baseline systemic symptoms and variables previously shown to predict $\mathrm{CO}$ VID-19 vaccine side effects (age, vaccine type, and prior COVID-19 infection [10]) accounted for significant variability in the reported number of vaccine side effects (Nagelkerke pseudo- $R^{2}=0.08$ ). We then added the psychosocial variables and observed this increased the variability accounted for in side effect reports (Nagelkerke pseudo- $\left.R^{2}=0.13\right)$. Specifically, vaccine side effect expectations (Wald $=11.21, p<0.001$ ), worry about COVID-19 (Wald $=6.87, p<0.01$ ), and depressive symptoms (Wald $=5.42, p<0.05)$ were each significant, with side effect expectations the strongest of these predictors. The psychosocial variables significantly improved the prediction of vaccine side effects compared to the demographic and clinical variables alone $\left(\chi^{2}=30.64, p<0.001\right)$. The psychosocial variables also remained significant predictors with baseline symptoms, age, vaccine type, and prior COVID-19 infection removed from the model.

We are the first to demonstrate a link between noceborelated factors and COVID-19 vaccine experienced side effects. Namely, pre-vaccine side effect expectations, worry about COVID-19, and depressive symptoms predicted COVID-19 vaccine side effects (i.e., headache, fatigue, pain at injection site), and did so beyond baseline symptomology, age, vaccine type, and prior COVID-19 infection. We identified nocebo-related predictors that are modifiable, and therefore could serve as critical points of intervention to reduce vaccine hesitancy and vaccine side effects. A number of strategies show promise in addressing negative expectations and reducing the nocebo effect, including thoughtful clinical information framing, reducing the negative impact of media coverage, and educating people about the nocebo effect [7]. Such strategies should be implemented at the individual and population level to improve the publics' experience of COVID-19 vaccines and ultimately, to curb vaccination hesitancy.

\section{Acknowledgement}

We thank Jon Elhai, PhD (Department of Psychology, University of Toledo, USA) for statistical consultation and Suzanne Helfer, PhD (Department of Psychology, Adrian College, USA) for comments on an earlier draft of the letter. 


\section{Conflict of Interest Statement}

The authors have no conflicts of interest to declare.

\section{Funding Sources}

This project was supported by a faculty research award provided to the first author by the Department of Psychology at the University of Toledo.

\section{Author Contributions}

Acquisition, analysis, and interpretation of data were done by Geers, Clemens, Faasse, and Jason, and the letter was drafted by Geers, Clemens, Faasse, and Colloca. All authors contributed to conceptualization, design, and to critical revision of the letter.

\section{References}

1 Cosci F, Guidi J. The role of illness behavior in the COVID-19 pandemic. Psychother Psychosom. 2021 Apr;90(3):156-9.

2 Cosci F, Fava GA. The clinical inadequacy of the DSM- 5 classification of somatic symptom and related disorders: an alternative trans-diagnostic model. CNS Spectr. 2016 Aug;21(4): 310-7.

3 Colloca L, Thomas S, Yin M, Haycock NR, Wang Y. Pain experience and mood disorders during the lockdown of the COVID-19 pandemic in the United States: an opportunistic study. Pain Rep. 2021 Sep 23;6(3):e958.
4 Solis Arce JS, Warren SS, Meriggi NF, Scacco A, McMurry N, Voors M, et al. COVID-19 vaccine acceptance and hesitancy in low- and middle-income countries. Nat Med. 2021 Jul; 27:1385-94.

5 The Economist/Yougov polls. https://today. yougov.com/topics/politics/articles-reports/2021/07/15/why-wont-americans-getvaccinated-poll-data (cited July 18, 2021).

6 Rief W. Fear of adverse effects and COVID-19 vaccine hesitancy: recommendations of the treatment expectation expert group. JAMA Health Forum. 2021 Apr;2(4):e210804.

7 Colloca L, Barsky AJ. Placebo and nocebo effects. N Engl J Med. 2020 Feb;382(6):554-61.
8 Polack FP, Thomas SJ, Kitchin N, Absalon J, Gurtman A, Lockhart S, et al. Safety and efficacy of the BNT162b2 mRNA COVID-19 vaccine. N Engl J Med. 2020 Dec;383(27): 2603-15.

9 Nagelkerke NJD. A note on a general definition of the coefficient of determination. Biometrika. 1991;78(3):691-2.

10 Menni C, Klaser K, May A, Polidori L, Capdevila J, Louca P, et al. Vaccine side-effects and SARS-CoV-2 infection after vaccination in users of the COVID Symptom Study app in the UK: a prospective observational study. Lancet Infect Dis. 2021 Apr;21(7):939-49. 